2 groups (41and 92 pts who achieved RR and didn't respectively). After 2 yrs the factors associated with RR achievement in pts with RA while non-biological DMARDs using were assessed.

Results: Before study, pts in both groups were comparable by all demographic, clinical and X-ray characteristics, frequency of DMARDs prescribed. They differed only in frequency of RF-positivity (39.0 and 57.6\% respectively in pts achieved $\mathrm{RR}$ and didn't, $\mathrm{p}<0.05$ ), aCCP-positivity ( 21.7 and $73.3 \%$ respectively, $\mathrm{p}<0.001$ ) and aCCP level $(6.06 \pm 0.88$ and $105.3 \pm 22.7 \mathrm{U} / \mathrm{ml}$ respectively, $p<0.001)$.

There was strong and moderate positive correlation between RR achivement and aCCP-negativity $(r=0.57, p<0.05)$, low aCCP level $(r=0.45, p<0.05)$ and $\triangle D A S 28$ $(r=0.35, p<0.05)$.

Results of multinomial logistic regression analyses (SPSS, V.22, IBM) showed that RF-negativity was the only independent predictor of $R R$ achievement $(B=3.14$, $p<0.05)$.

$41.7 \%$ pts in RR achieved clinical remission by DAS28 as well. Only $19.7 \%$ pts achieved clinical remission without $R R(p<0.05$ vs pts achieved clinical and $R R)$ and $12.5 \%$ pts achieved RR without clinical response (DAS28 $>5.1, \triangle \mathrm{DAS} 28 \leq 1.2$ or any value of DAS28 with $\triangle \mathrm{DAS} 28<0.6 ; \mathrm{p}<0.01)$.

After 2 years of treatment the mean value of $\triangle \mathrm{DAS} 28$ in comparison groups (with and without $R R$ ) did not differ $(2.79 \pm 0.28$ vs $2.33 \pm 0.17$ respectively) so there was a discrepancy between clinical and radiological outcome.

Conclusions: Baseline predictors associated with achieving of radiographic remission in pts with RA are RF- and aCCP-negativity, low level of aCCP, but independent predictor of radiographic remission achievement is the only RF-negativity.

The discrepancy between the frequency of clinical and radiographic remission achievement in RA patients is observed.

After 2 yrs of non-biological DMARDs treatment $41.7 \%$ pts with RA achieves radiographic and clinical remission at the same time.

Disclosure of Interest: None declared

DOI: 10.1136/annrheumdis-2017-eular.2682

\section{THU0095 IMPACT OF ULTRASOUND-DETECTED TENOSYNOVITIS AND SYNOVITIS ON DISEASE FLARE, DISABILITY AND RADIOGRAPHIC PROGRESSION IN PATIENTS WITH RA IN CLINICAL REMISSION: RESULTS OF THE STARTER STUDY}

G. Sakellariou ${ }^{1}$, G. Filippou ${ }^{2}$, E. Bellis ${ }^{3}$, C.A. Scirè ${ }^{4}$, G. Carrara ${ }^{5}$, A. Iagnocco $^{6}$ on behalf of STARTER study group and US Study Group of the Italian Society for Rheumatology. ${ }^{1}$ IRCCS Policlinico San Matteo Foundation, Pavia; ${ }^{2}$ University of Siena, Siena; ${ }^{3}$ Ospedale Mauriziano, Torino; ${ }^{4}$ Italian Society for Rheumatology, Milan; ${ }^{5}$ Italian Society for Rheumatology, Milano; ${ }^{6}$ Università degli Studi di Torino, Torino, Italy

Background: In patients with RA in remission subclinical ultrasonographic (US) synovitis (-S) relates to flare and radiographic progression. The impact of tenosynovitis (-T) on flare, disability and radiographic progression is not known.

Objectives: To evaluate the predictive role of US-detected tenosynovitis and synovitis in RA patients in remission on flare, disability and radiographic progression over 12-months.

Methods: STARTER is a multicentre cohort study of the US Study Group of the Italian Society for Rheumatology. Participants were selected on the basis of a reliability exercise and the availability of high-end equipment with high frequencies probes. Patients with RA in remission underwent clinical and US evaluation. US -T and -S were assessed categorically by Grey Scale (GS) and power Doppler (PD) at 11 joints, extensor and flexor tendons in both hands. Patients were assesed at baseline, 6 and 12 months. The primary outcome was flare within 12 months (defined as increase in DAS28 $>1.2$ or $>0.6$ if final DAS28 $>3.2$ ). The secondary outcomes were progression of disability (increase $\geq 2.3$ in the Health Assessment Questionnaire (HAQ)) and radiographic progression (increase in the total Sharp van Der Hejide score (SHS) $\geq 4.3$ ) at 12 months. Logistic models were used to

Table 1

\begin{tabular}{lcc}
\hline & OR $(95 \% \mathrm{Cl})$ & Adj OR $(95 \% \mathrm{Cl})$ \\
\hline$\Delta$ DAS28 $>1.2$ or $>0.6$ if final & \\
PDS $28>3.2$ & \\
PD-S & $0.59(0.16,2.15)$ & $0.47(0.12,1.82)$ \\
PD-T + PD-S & $1.64(0.93,2.90)$ & $1.59(0.86,2.92)$ \\
GS-T & $2.75(1.45,5.20)$ & $2.09(1.06,4.13)$ \\
GS-S & $1.59(0.53,4.72)$ & $1.37(0.42,4.41)$ \\
GS-T + GS-S & $2.18(0.97,4.92)$ & $1.88(0.79,4.46)$ \\
$\Delta$ HAQ $\geq 0.23$ & $2.88(1.34,6.14)$ & $2.25(1.00,4.06)$ \\
PD-T & & \\
PD-S & $0.64(0.13,3.03)$ & $0.81(0.16,4.01)$ \\
PD-T + PD-S & $0.79(0.37,1.68)$ & $0.71(0.32,1.54)$ \\
GS-T & $1.49(0.68,3.26)$ & $1.33(0.57,3.11)$ \\
GS-S & $0.77(0.22,2.73)$ & $0.64(0.17,2.45)$ \\
GS-T + GS-S & $0.83(0.34,1.97)$ & $0.61(0.24,1.54)$ \\
$\Delta$ Total SHS $\geq 4.3$ & $0.93(0.42,2.02)$ & $0.64(0.27,1.53)$ \\
PD-T & & \\
PD-S & $0.82(0.09,7.37)$ & $0.54(0.05,5.76)$ \\
PD-T + PD-S & $1.61(0.71,3.64)$ & $1.60(0.68,3.79)$ \\
GS-T & $1.53(0.60,3.93)$ & $1.09(0.38,3.11)$ \\
GS-S & $0.78(0.08,7.88)$ & $0.67(0.06,7.50)$ \\
GS-T + GS-S & $2.38(0.72,7.84)$ & $1.78(0.49,6.48)$ \\
\hline
\end{tabular}

measure the relationship between GS-T/-S, PD-T/-S and outcomes, results were presented as odds ratios (OR) and 95\% confidence interval $(\mathrm{Cl})$, adjusted for pre-specified confounders.

Results: 361 patients $(72.3 \%$ f, mean age (sd) 56.1 (13.3), median disease duration (IQR) 7.1 years (3.6-13.5)) were included, complete radiographs were available for $189 / 361$ (52.3\%) patients. 98/326 (30.6\%) patients had a flare within 12 months, 70/340 (20.59\%) had an increase in HAQ and 39/189 (20.6\%) radiographic progression. Results are presented in Table 1. Flare was predicted only if $-T$ and $-S$, assessed by both GS and PD, were concurrently present, while both $-T$ and-S and their combination did not predict $\mathrm{HAQ}$ or SHS progression.

Conclusions: In patients with RA in clinical remission, US-detected synovial and tenosynovial inflammation relates to the risk of flare, while in a short term follow-up the effect on disability and radiographic progression is limited. These results might have been influenced by the short follow-up and limited power for secondary outcomes. US might integrate the clinical management of RA patients in clinical remission.

Disclosure of Interest: None declared

DOI: 10.1136/annrheumdis-2017-eular.4774

\section{THU0096 MULTI-BIOMARKER DISEASE ACTIVITY (MBDA) IS ASSOCIATED WITH THE PROGRESSION OF STRUCTURAL BONE DAMAGE IN RHEUMATOID ARTHRITIS PATIENTS IN REMISSION}

G. Schett ${ }^{1}$, S. Finzel ${ }^{2}$, M. Hagen ${ }^{1}$, M. Englbrecht ${ }^{1}$, J. Haschka ${ }^{3}$,

C. Figuereido ${ }^{4}$, J. Fogagnolo Cobra ${ }^{4}$, J. Rech ${ }^{1}$. ${ }^{1}$ University of

Erlangen-Nuremberg, Erlangen; ${ }^{2}$ University Medical Center Freiburg,

Rheumatology and Clinical Immunology, Freiburg, Germany; ${ }^{3}$ St.Vincent

Hospital, Vinforce Study Group, Medical University of Vienna, Vienna, Austria;

${ }^{4}$ Institutio de Rheumatologia, Sao Paolo, Brazil

Background: Due to an increasing number and an earlier use of effective disease modifying anti-rheumatic drug (DMARD) therapy a growing number of rheumatoid arthritis (RA) patients reaches a state of clinical remission of disease. Whether clinical remission completely protects from structural bone damage, however, is still a matter of debate as conventional radiographic methods have their limitation in the sensitivity to characterize bone damage. Furthermore, residual activity of inflammation associated with the generation of inflammatory markers may characterize a subset of RA patients in remission, associated with higher prevalence and/or progression of bone damage.

Objectives: To test whether residual systemic inflammation is associated with structural bone damage and the progression of structural bone damage in RA patients in sustained remission.

Methods: RA patients (i) fulfilling the 2010 EULAR/ACR classification criteria of RA, (ii) having a positive anti-citrullinated protein antibody (ACPA) status and (iii) being in DAS28-ESR remission for 6 months were included. High-resolution peripheral quantitative computed tomography (HR-pQCT) of the right hand was done at baseline and after 1year. Erosion numbers and erosion volumes were assessed in the metacarpophalangeal joints. Vectra-DA tests measuring the serum levels of twelve different inflammation markers (CRP, SAA, IL-6, TNFR1, MMP-1, MMP-3, EGF, VEGF-A, VCAM-1, YKL-40, leptin and resistin) were performed in the baseline samples. MBDA score was calculated according to previously defined algorithms with low MDBA score defined as $<30$ units and moderate to high scores as $\geq 30$ units (1).

Results: $100 \mathrm{ACPA}+\mathrm{RA}$ patients in sustained remission were investigate (mean \pm SD age: $57 \pm 14$ ys; disease duration: $4.8 \pm 4.9$ ys; DAS28: $1.7 \pm 0.5 ; 63 \%$ females; $100 \%$ MTX treatment, $38 \%$ MTX+TNFi treatment). 65 patients had low $(<30), 25$ patients has moderate $(>30-44$ and 10 patients had high $(>44)$ MBDA scores. Patients in the different MBDA categories had similar age, sex, disease duration, DAS28 scores and DMARD treatment. Baseline HR-pQCT analysis showed that erosion numbers and volumes were significantly $(\mathrm{p}<0.001)$ higher in patients with high MBDA scores. Higher erosion numbers $(>10)$ and larger erosions $\left(>10 \mathrm{~mm}^{3}\right)$ were exclusively found in patients with moderate to high MBDA scores. MBDA scores were correlated to erosion numbers $(p<0.001)$ and volumes $(p=0.0018)$. Longitudinal analysis showed significant progression of erosions only in patients with high MBDA scores. Furthermore, progression $\left(>5 \mathrm{~mm}^{3}\right.$ increase in volume) over 1 year was confined to methotrexate treated patients, while tumor necrosis factor inhibitor treated patients were protected from progression even in case of moderate to high MBDA scores. However there were only 4 patients treated with TNFi that had a high MBDA score.

Conclusions: These data show that residual disease activity assessed by MBDA score is associated with structural damage and progression of structural damage in RA patients in sustained remission.

\section{References:}

[1] Curtis JR, van der Helm-van Mil AH, Knevel R, et al.Validation of a novel multi-biomarker test to assess rheumatoid arthritis disease activity. Arthritis Care Res 2012;64:1794-803.

Disclosure of Interest: None declared

DOI: 10.1136/annrheumdis-2017-eular.6227 